\title{
Despeckling of Images using Weiner Filter and Hybrid Median Filter
}

\author{
Sarita Gupta \\ M.Tech (CS) \\ PSIT, Kanpur \\ Computer Science Department
}

\author{
Saurabh Gupta \\ Assistant Professor \\ PSIT, Kanpur \\ Computer Science Department
}

\begin{abstract}
Images are a crucial part of everyone's life in this modern world. The increase in the number of these has led to a need of better results like better quality, better diagnosis of medical problems etc. Reducing noise from the images is a challenge for the researchers in digital image processing. Several approaches are there for noise reduction. Generally speckle noise is commonly found in the synthetic aperture. This research proposes techniques for the removal of speckle noise from the images. In our proposed method we use medical images and denoise it to remove speckle noise. Weiner filter in combination with hybrid median filter is proposed for the problem in hand. It gives better results for the denoising of the speckle noise corrupted image.
\end{abstract}

\section{General Terms}

Weiner filter, hybrid filter, speckle noise, DWT

\section{Keywords}

Weiner Filter, Dspeckle Noise, Kuan Filter, Hybrid Median Filter, Image Denoising

\section{INTRODUCTION}

All coherent imaging systems like laser, acoustic and SAR imagery are affected by speckle noise as a common phenomenon. The source of this noise is attributed to random interference between the coherent returns issued from the numerous scatters present on a surface, on the scale of a wavelength of the incident radar wave. Speckle noise is often an undesirable, especially for ATR systems. Thus, speckle filtering turns out to be a critical pre-processing step for detection or classification optimization.

\subsection{Speckle statistics}

Fully developed speckle has the characteristics of a random multiplicative] noise. Under the assumption that the real and imaginary parts respectively the so-called in-phase (denoted I) and quadrature (denoted Q) components of a complex radar image of the speckle have zero-mean Gaussian density, noise intensity can be shown to follow a Gamma distribution (which reduces to an exponential distribution for single-look images). The mean-to-standard-deviation ratio (a measure of the signalto-noise ratio) of such a distribution satisfies

$$
\left(\frac{\text { mean }}{\text { standard deviation }}\right)^{2}=L=\text { constant }
$$

\section{PROPOSED METHODOLOGY}

The proposed method deals with removal of speckle noise from images. The method uses wiener filter for removal of course speckle noise. Firstly we find logarithmic transform of the noisy image. This step is performed because speckle noise is a multiplicative noise. Logarithmic transform converts this into additive noise. The discrete wavelet transform of this image is found. This gives us some detail and approximation coefficients. We apply wiener filter on the detail coefficients. Further we find the inverse discrete wavelet transform. The resultant image is filtered using hybrid median filter which is efficient enough to remove the speckle noise. Finally exponential transform is applied to recover the denoised image.
2.1 Flow of Algorithm
1) Input image
2) Add noise
3) Apply Logarithmic transform
4) DWT haar wavelet
5) Denoise using wiener filter
6) Inverse DWT
7) Denoise using hybrid median filter
8) Apply Inverse Logarithmic transform
9) Output image

\section{RESULTS}

The algorithm discussed has been implemented in matlab and tested on Intel i3 processor with 4GB. The images taken for testing purpose are of size $512 \times 512$ pixels. The proposed method runs robustly denoising the speckle noise in the given noisy image. The result of few images can be seen in figure below
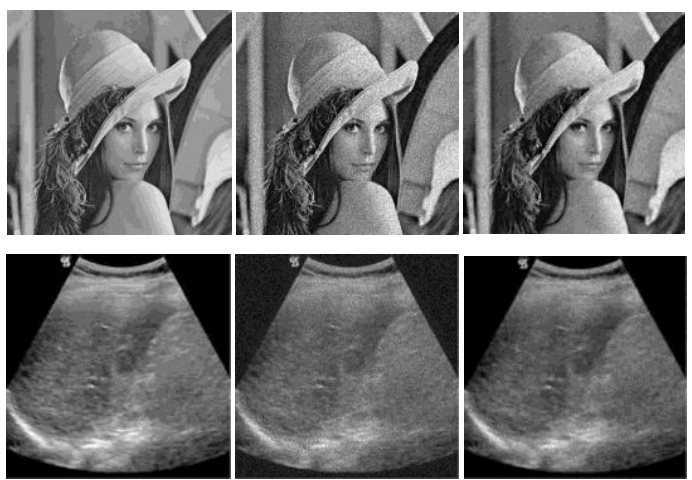

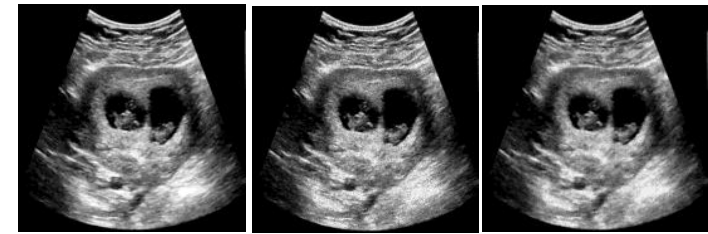

Figure, The image on the left is the noise free image, the image in the middle is the noisy image and the image in the right is the denoised image.

The images in the figure below shows have been presented to show the effect of speckle noise. The noise free image is taken and speckle noise is added to it. It is then denoised using our proposed technique. The denoised image is very much similar to the noise free image.
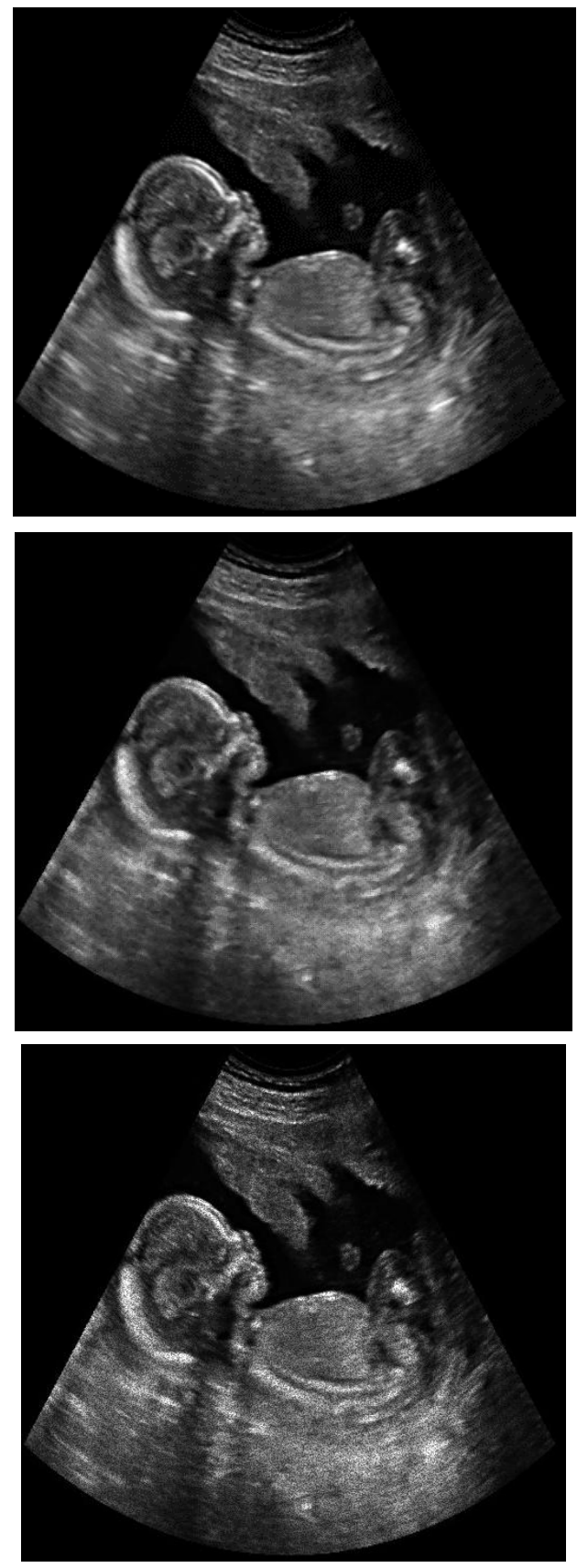

Figure, Image in the 1 st row is the noise free image, 2 nd row is the denoised image and image in the 3rd row is the noisy image.
The results of the base paper and the proposed algorithm has been presented in table 1 . The table shows that there is an increase in approximately 5db in PSNR and the MSE value has decreased by almost 3 times. Also it can seen that the time required for the execution of the algorithm takes approximately $5.4 \mathrm{sec}$. whereas the proposed algorithm takes approximately $1.68 \mathrm{sec}$. Thus the proposed algorithm is faster than the base method by 3.2 times.

Table 1

\begin{tabular}{|c|c|c|c|c|c|c|c|c|}
\hline $\begin{array}{l}\text { S. } \\
\text { No }\end{array}$ & $\begin{array}{c}\text { PSN } \\
\text { R } \\
\text { (Bas } \\
\text { e) }\end{array}$ & $\begin{array}{c}\text { MS } \\
\text { E } \\
(\text { Ba } \\
\text { se })\end{array}$ & $\begin{array}{c}\text { Ma } \\
\text { x } \\
\text { Err } \\
\text { or } \\
\text { (Ba } \\
\text { se) } \\
\end{array}$ & $\begin{array}{c}\text { Tim } \\
\text { e } \\
(\text { Bas } \\
\text { e- } \\
\text { sec) }\end{array}$ & $\begin{array}{c}\text { PSN } \\
\text { R }\end{array}$ & $\begin{array}{c}\text { MS } \\
\text { E }\end{array}$ & $\begin{array}{c}\text { Ma } \\
\text { X } \\
\text { Err } \\
\text { or }\end{array}$ & $\begin{array}{c}\text { Tim } \\
\text { e } \\
\text { (sec) }\end{array}$ \\
\hline 1 & $\begin{array}{r}24.7 \\
576 \\
\end{array}$ & $\begin{array}{c}0.0 \\
134 \\
\end{array}$ & $\begin{array}{r}0.3 \\
859\end{array}$ & $\begin{array}{r}5.73 \\
9101 \\
\end{array}$ & $\begin{array}{l}28.8 \\
229\end{array}$ & $\begin{array}{r}0.0 \\
052 \\
\end{array}$ & $\begin{array}{r}0.3 \\
851 \\
\end{array}$ & $\begin{array}{l}1.66 \\
4350\end{array}$ \\
\hline 2 & $\begin{array}{l}23.1 \\
862 \\
\end{array}$ & $\begin{array}{c}0.0 \\
303 \\
\end{array}$ & $\begin{array}{l}0.3 \\
644 \\
\end{array}$ & $\begin{array}{r}5.38 \\
7182 \\
\end{array}$ & $\begin{array}{l}26.7 \\
995 \\
\end{array}$ & $\begin{array}{c}0.0 \\
132 \\
\end{array}$ & $\begin{array}{c}0.2 \\
861 \\
\end{array}$ & $\begin{array}{r}1.74 \\
5610 \\
\end{array}$ \\
\hline 3 & $\begin{array}{l}24.2 \\
868 \\
\end{array}$ & $\begin{array}{r}0.0 \\
323 \\
\end{array}$ & $\begin{array}{r}0.4 \\
953 \\
\end{array}$ & $\begin{array}{l}5.42 \\
1320 \\
\end{array}$ & $\begin{array}{l}30.5 \\
843 \\
\end{array}$ & $\begin{array}{c}0.0 \\
076 \\
\end{array}$ & $\begin{array}{c}0.2 \\
530 \\
\end{array}$ & $\begin{array}{r}1.68 \\
9274 \\
\end{array}$ \\
\hline 4 & $\begin{array}{l}27.6 \\
525 \\
\end{array}$ & $\begin{array}{l}0.0 \\
295 \\
\end{array}$ & $\begin{array}{l}0.4 \\
435\end{array}$ & $\begin{array}{r}5.32 \\
3250 \\
\end{array}$ & $\begin{array}{l}32.7 \\
268 \\
\end{array}$ & $\begin{array}{c}0.0 \\
092\end{array}$ & $\begin{array}{c}0.3 \\
419 \\
\end{array}$ & $\begin{array}{c}1.66 \\
7756 \\
\end{array}$ \\
\hline
\end{tabular}

The comparison of the statistics of noisy image and the denoised image has been shown in table 2 . We can see that the proposed algorithm is increasing the PSNR value by almost 6 $\mathrm{db}$ and MSE value has reduced by 3 times.

Table 2

\begin{tabular}{|c|c|c|c|c|c|c|}
\hline $\begin{array}{c}\text { S.No } \\
\cdot\end{array}$ & $\begin{array}{c}\text { PSNR } \\
\text { (Noise } \\
\text { ) }\end{array}$ & $\begin{array}{c}\text { MSE } \\
\text { (Noise } \\
\text { ) }\end{array}$ & $\begin{array}{c}\text { Max } \\
\text { Error } \\
\text { (Noise } \\
\text { ) }\end{array}$ & PSNR & MSE & $\begin{array}{c}\text { Max } \\
\text { Erro } \\
\mathbf{r}\end{array}$ \\
\hline $\mathbf{1}$ & $\begin{array}{c}21.186 \\
6\end{array}$ & 0.030 & 0.242 & 28.822 & 0.005 & 0.385 \\
& 4 & 5 & 9 & 2 & 1 \\
\hline $\mathbf{2}$ & 21.382 & 0.045 & 0.411 & 26.799 & 0.013 & 0.286 \\
& 9 & 8 & 8 & 5 & 2 & 1 \\
\hline $\mathbf{3}$ & 24.814 & 0.028 & 0.298 & 30.584 & 0.007 & 0.253 \\
& 5 & 6 & 0 & 3 & 6 & 0 \\
\hline $\mathbf{4}$ & 27.595 & 0.029 & 0.286 & 32.726 & 0.009 & 0.341 \\
& 4 & 9 & 3 & 8 & 2 & 9 \\
\hline
\end{tabular}

\section{CONCLUSION}

The proposed algorithm is robust in performing image enhancement dealing with removal of speckle image. The results show that the proposed method is better than []. The algorithm proves an increase in the PSNR value by almost $6 \mathrm{db}$ and MSE value has reduced by 3 times. Thus the proposed method can be used for image enhancement for removal of speckle noise from images in applications.

\section{REFERENCES}

[1] Speckle Filtering of SAR Images - A Comparative Study Between Complex-Wavelet-Based and Standard Filters L. Gagnon and A. Jouan D_epartement de R\&D, Lockheed Martin Canada, 6111 Ave. Royalmount, Montr_eal(Qu_ebec), H4P 1K6, CANADA

[2] Pizurica, A., Philips, W., Lemahieu, I., and Acheroy, M.: 'A versatile wavelet domain noise filtration technique for medical imaging', IEEE Trans. Med. Imaging, 2003, 22, (3), pp. 323-331 
[3] Bhuiyan, M.I.H., Ahmad, M.O., and Swamy, M.N.S.: 'Spatially adaptive thresholding in wavelet domain for speckling of ultrasound images', IET Image Proc., 2009, 3, (3), pp. 147-162

[4] Hao, X., Gao, S., and Gao, X.: 'A novel multiscale nonlinear thresholding method for ultrasonic speckle suppressing', IEEE Trans.Med. Imaging, 1999, 18, (9), pp. 787-794

[5] Adib Akl, Charles Yaacoub, 'A HYBRID WAVELET SPATIAL DENOISING FILTER', DSP2013, 978-14673-5807-1/13

[6] Adib Akl, Kamal Tabbara, Charles Yaacoub.' EDGEBASED AUTOMATIC PARAMETER SELECTION
FOR SUB-OPTIMAL IMAGE DESPECKLING USING KUAN FILTER', 2012 Eighth International Conference on Signal Image Technology and Internet Based SystemsFeature and Neural based Classification," International Joint Conference on Neural Networks, 2006.

[7] Lal Chandra, Puja Lal, Raju Gupta, Arun Tayal,Dinesh Ganotra: Improved adaptive binarization technique for document image analysis. VISAPP (1) 2007: 317-321.

[8] Ved Prakash Agnihotri, "Offline Handwritten Devanagari Script Recognition", I.J. Information Technology and Computer Science, 2012, 8, 37-42 